\title{
Prognostic Implications of the Complement Protein C1q in Gliomas
}

\begin{abstract}
Alessandro Mangogna ${ }^{1 \dagger}$, Beatrice Belmonte ${ }^{2 \dagger}$, Chiara Agostinis ${ }^{3}$, Paola Zacchi ${ }^{1 *}$, Domenico Gerardo lacopino ${ }^{4}$, Anna Martorana ${ }^{5}$, Vito Rodolico ${ }^{5}$, Deborah Bonazza ${ }^{6}$, Fabrizio Zanconati ${ }^{6}$, Uday Kishore ${ }^{7}$ and Roberta Bulla ${ }^{1}$
\end{abstract}

\begin{abstract}
${ }^{1}$ Department of Life Sciences, University of Trieste, Trieste, Italy, ${ }^{2}$ Human Pathology Section, Tumour Immunology Unit, Department of Health Sciences, University of Palermo, Palermo, Italy, ${ }^{3}$ Institute for Maternal and Child Health, IRCCS (Istituto di Ricovero e Cura a Carattere Scientifico) Burlo Garofolo, Trieste, Italy, ${ }^{4}$ Neurosurgical Unit, Department of Experimental Biomedicine and Clinical Neuroscience, University Hospital, Paolo Giaccone, University of Palermo, Palermo, Italy, ${ }^{5}$ Department of Health Promotion, Mother and Child Care, Internal Medicine and Medical Specialties, University of Palermo, Palermo, Italy, ${ }^{6}$ Department of Medical, Surgical and Health Science, University of Trieste, Trieste, Italy, ${ }^{7}$ Biosciences, College of Health and Life Sciences, Brunel University London, London, United Kingdom
\end{abstract}

The contribution of the complement system in the pathophysiology of brain cancers has been recently considered in light of its well-known involvement in carcinogenesis.

OPEN ACCESS

Edited by:

Robert Braidwood Sim, University of Oxford, United Kingdom

Reviewed by:

Lubka T. Roumenina,

INSERM U1138 Centre de Recherche des Cordeliers, France Kenneth Reid,

University of Oxford, United Kingdom

*Correspondence:

Paola Zacchi

pzacchi@units.it

tThese authors have contributed equally to this work

Specialty section:

This article was submitted to

Molecular Innate Immunity,

a section of the journal

Frontiers in Immunology

Received: 21 June 2019 Accepted: 20 September 2019

Published: 10 October 2019

Citation:

Mangogna A, Belmonte $B$ Agostinis C, Zacchi P, lacopino DG, Martorana A, Rodolico V, Bonazza D,

Zanconati F, Kishore $U$ and Bulla $R$ (2019) Prognostic Implications of the Complement Protein C1q in Gliomas.

Front. Immunol. 10:2366. doi: 10.3389/fimmu.2019.02366
Complement system represents an important component of the inflammatory response, which acts as a functional bridge between the innate and adaptive immune response. $\mathrm{C} 1 \mathrm{q}$, the first recognition subcomponent of the complement classical pathway, has recently been shown to be involved in a range of pathophysiological functions that are not dependent on complement activation. $\mathrm{C} 1 \mathrm{q}$ is expressed in the microenvironment of various types of human tumors, including melanoma, prostate, mesothelioma, and ovarian cancers, where it can exert a protective or a harmful effect on cancer progression. Despite local synthesis of $\mathrm{C} 1 \mathrm{q}$ in the central nervous system, the involvement of $\mathrm{C} 1 \mathrm{q}$ in glioma pathogenesis has been poorly investigated. We, therefore, performed a bioinformatics analysis, using Oncomine dataset and UALCAN database in order to assess whether the expression of the genes encoding for the three chains of $\mathrm{C} 1 \mathrm{q}$ $(C 1 q A, C 1 q B$, and $C 1 q C)$ could serve as a potential prognostic marker for gliomas. The obtained results were then validated using an independent glioma cohort from the Chinese Glioma Genome Atlas datasets. Our bioinformatics analysis, coupled with immunohistochemistry and fluorescence microscopy, appears to suggest a positive correlation between higher levels of $\mathrm{C} 1 \mathrm{q}$ expression and unfavorable prognosis in a diverse grade of gliomas.

Keywords: gliomas, C1q complement, bioinformatics analysis, survival probability, prognostic significance of C1q

\section{INTRODUCTION}

The complement protein $\mathrm{Clq}$ represents the recognition subcomponent of the complement classical pathway, which is responsible for clearing immune complexes and invading pathogens. Its association with $\mathrm{C} 1 \mathrm{r}$ and $\mathrm{C} 1 \mathrm{~s}$, following ligand recognition, triggers complement activation $(1,2) . \mathrm{Clq}$ is characterized by a typical tulip-like overall structure, assembled from 18 polypeptide chains of three different types, A $(28 \mathrm{kDa}), \mathrm{B}(25 \mathrm{kDa})$, and $\mathrm{C}(24 \mathrm{kDa})$, each having an $\mathrm{N}$-terminal collagen-like domain and a C-terminal globular (gClq) domain 
$(3,4)$. The gClq domain, which is the ligand recognition region of $\mathrm{Clq}$, has a heterotrimeric structure, being composed of $\mathrm{C}$ terminal ends of A, B, and C chains (5). In addition to binding IgG and IgM containing immune complexes and activating the complement classical pathway, there is emerging evidence to suggest that $\mathrm{Clq}$ plays crucial roles in several processes that are independent of complement activation, such as placentation (6), angiogenesis (7), autoimmunity $(8,9)$, and carcinogenesis $(9,10) . \mathrm{Clq}$ is highly expressed in the microenvironment of various types of human tumors $(10,11)$ where it can exert either a protective or a detrimental effect on the tumor growth. In prostate cancer cells, $\mathrm{C} 1 \mathrm{q}$ was recently shown to induce apoptosis by activating the tumor suppressor WOX1 (12), thus acting as an anti-tumor humoral factor. In ovarian cancer, $\mathrm{C} 1 \mathrm{q}$ has been shown to induce apoptosis in a representative SKOV3 cell line via activation of TNF- $\alpha$, upregulation of Fas, and downregulation of mammalian target of rapamycin, RICTOR, and RAPTOR survival pathways (13). In a BALBneuT mouse model of mammary carcinomas, $\mathrm{C} 1 \mathrm{q}$ was shown to have a protective role against cancer progression (14). However, C1q can promote adhesion, migration, and proliferation of primary cells derived from malignant pleural mesothelioma patients, a relatively rare disease associated with exposure to asbestos (11). This dichotomous role of $\mathrm{Clq}$ has been further highlighted by a bioinformatics analysis involving several types of carcinomas (15).

The importance of $\mathrm{Clq}$ in the pathophysiology of the central nervous system (CNS) has been an area of intense research in the last two decades. In a healthy brain, $\mathrm{Clq}$ promotes synapse elimination required for fine circuitry refinement during CNS development (16). C1q activities, unrelated to complement activation, were shown to support neuronal survival and neurite outgrowth in vitro and protect against $\beta$-amyloid-mediated neurotoxicity (17). C1q can also interact with abnormal protein aggregates, such as $\beta \mathrm{A} 1-42$, thus favoring neurodegenerative diseases progression (18).

Since C1q and other complement components can be locally produced within the CNS by microglia and astrocytes, it is likely that $\mathrm{C} 1 \mathrm{q}$ has involvement in primary brain tumor pathophysiology (19). Brain malignancies arise from cells of the CNS and are classified according to the tissue of phylogenetic origin. Gliomas represent the most common and aggressive form of brain tumors in adults; they are derived from glial or precursor cells (20). These are a heterogeneous group of diseases with multiple subtypes $(20,21)$. Glioblastoma multiforme (GBM) is the most common and fatal form of the primary brain tumor, accounting for approximately $60 \%$ of all glioma cases (22), whereas low-grade gliomas (LGGs) are the second most common type of glioma in adults $(\sim 30 \%)(22)$.

In GBM tumor specimens, the presence of $\mathrm{C} 1 \mathrm{q}$ does not correlate with CD45 positive leukocytic infiltration (23). Interestingly, C1q appeared to be highly concentrated around the malignant cells and the necrotic debris. Moreover, the serum concentration of $\mathrm{Clq}$, together with critical components of the lectin and alternative complement pathways, appeared significantly increased in GBM patients as compared to healthy controls (23), thus indicating a role for complement activation in the pathogenesis of the GBM.
In the current study, we performed a bioinformatics analysis aimed at investigating whether $\mathrm{C} 1 \mathrm{q}$ can serve as a potential prognostic marker for gliomas.

\section{MATERIALS AND METHODS \\ Oncomine Database Analysis}

The expression levels of $C 1 q A, C 1 q B$, and $C 1 q C$ genes in gliomas were analyzed using Oncomine (www.oncomine.org), a cancer microarray database and web-based data mining platform for new discovery from genome-wide expression analyses $(24,25)$. We compared the differences in mRNA levels between normal tissue and cancer. The mRNA expression level in neoplastic tissues, compared to the healthy tissues, was obtained as the parameters of $P<0.05$, fold change $>2$, and gene ranking in the top $10 \%$. Information about the datasets used in this study is summarized in Table $\mathbf{1 .}$

\section{UALCAN and CGGA Database Analysis}

UALCAN (http://ualcan.path.uab.edu) is a web resource for analyzing cancer transcriptome data, which estimates the effect of gene expression level on the patient survival (26). In addition to the gene expression variation across tumor samples, genelevel correlations with patient survival also feature in UALCAN. Available genomics data from "The Cancer Genome Atlas" (TCGA) project was used for Kaplan-Meier survival analysis to generate survival probability plots (26). The prognostic significance of $C 1 q A, C 1 q B$, and $C 1 q C$ expression and survival in gliomas were analyzed by UALCAN. The hazard ratio with 95\% confidence intervals and logrank $p$-value were also computed.

The Chinese Glioma Genome Atlas (CGGA) (http://www. cgga.org.cn) is a user-friendly web application for data storage and analysis exploring brain tumors datasets from Chinese cohorts (Table 1) (27). Analyze tool of CGGA was used to browse $C 1 q A, C 1 q B$, and $C 1 q C$ mRNA expression profile and to perform survival analysis in specific glioma subtype. The hazard ratio with 95\% confidence intervals and logrank $p$-value were also computed.

\section{Statistical Analysis}

Survival curves were generated by UALCAN and CGGA. All results are displayed with $p$-values from a log-rank test. $P$ values $<0.05$ were considered significant. Similarly, in the case of Oncomine, the program provided the statistical significance of data ( $P$-values).

\section{Immunostaining}

Tissue samples, derived from the neuroepithelial tumors with astrocytic differentiation, presenting different grades (gradeII and -III to GBM, grade-IV), were collected from glioma patients, after informed consent following approval of the ethical considerations by the Institutional Board of the University Hospital of Trieste, Italy.

Gliomas tissue specimens (five patients for each glioma grade, Department of human pathology of the University Hospital of Cattinara, Trieste, Italy) were fixed in $10 \% \mathrm{v} / \mathrm{v}$ buffered formalin and paraffin embedded. For immunostaining, $4 \mu \mathrm{m}$-thick tissue 
TABLE 1 | Characteristics of the datasets used in bioinformatics analysis.

\begin{tabular}{|c|c|c|}
\hline Datasets & Study description & Experiment type \\
\hline Sun brain & $\begin{array}{l}\text { One hundred fifty-seven (157) brain and CNS tumors and } 23 \text { normal brain samples were analyzed on Affymetrix U133 Plus } \\
2.0 \text { microarrays. Sample data includes type, grade, and sample name. Corresponding DNA copy number data is available } \\
\text { in Kotliarov Brain }\end{array}$ & mRNA \\
\hline French brain & $\begin{array}{l}\text { Twenty-three (23) anaplastic oligodendroglioma, } 4 \text { anaplastic oligoastrocytoma, and } 6 \text { normal brain samples were analyzed } \\
\text { on Affymetrix U133 Plus } 2.0 \text { microarrays. Sample data includes 10q loss of heterozygosity, 19q loss of heterozygosity, } 1 p \\
\text { loss of heterozygosity, age, chemotherapy response, EGFR amplification, sex, survival after diagnosis, survival after surgical } \\
\text { resection, and therapy }\end{array}$ & mRNA \\
\hline TCGA brain & $\begin{array}{l}\text { Five hundred forty-seven (547) glioblastoma and } 10 \text { normal brain samples were analyzed. Sample data includes age, sex, } \\
\text { survival, and others. This dataset consists of Level } 2 \text { (processed) data from the TCGA data portal. Corresponding DNA copy } \\
\text { number data is available in TCGA Brain } 2\end{array}$ & mRNA \\
\hline CGGA brain & $\begin{array}{l}\text { The CGGA RNA sequence dataset consisted of } 325 \text { samples, including } 109 \text { grade-II samples, } 72 \text { grade-III samples and } \\
144 \text { grade-IV samples. Of the } 144 \text { GBM samples, } 6 \text { samples were lost to follow-up; therefore, } 138 \text { samples were included } \\
\text { in the survival analysis. The patients with GBM were followed up every } 3 \text { months }\end{array}$ & mRNA \\
\hline Rickman brain & $\begin{array}{l}\text { Forty-five (45) astrocytoma and } 6 \text { normal temporal lobe samples were analyzed on Affymetrix HuGeneFL microarrays. } \\
\text { Sample data include type and grade }\end{array}$ & mRNA \\
\hline Bredel brain 2 & $\begin{array}{l}\text { Fifty (50) brain CNS carcinoma samples and } 4 \text { normal brain samples were analyzed on cDNA microarrays. Sample data } \\
\text { includes disease type }\end{array}$ & mRNA \\
\hline Liang brain & $\begin{array}{l}\text { Thirty (29) glioblastoma, } 3 \text { mixed astrocytoma-oligodendroglioma, } 2 \text { oligodendroglioma, } 2 \text { normal brain, and } 1 \text { normal } \\
\text { cerebellum sample were analyzed on cDNA microarrays. Sample data includes type, age, location, primary/recurrent, sex, } \\
\text { and survival }\end{array}$ & mRNA \\
\hline
\end{tabular}

sections were de-waxed with two changes of xylene, $10 \mathrm{~min}$ each. Slides were then transferred to $100 \%$ alcohol, for two changes, 10 min each, and once through 95 and $70 \%$ alcohol respectively, for $5 \mathrm{~min}$ each. Finally, they were rinsed in de-ionized water, twice for $3 \mathrm{~min}$ each. The antigen unmasking technique was performed using Novocastra Epitope Retrieval Solutions pH9 EDTA-based buffer in thermostatic bath at $98^{\circ} \mathrm{C}$ for $30 \mathrm{~min}(28)$. Sections were brought to room temperature and washed in PBS. Subsequently, the neutralization of the endogenous peroxidase with $3 \% \mathrm{v} / \mathrm{v}_{2} \mathrm{O}_{2}$ and $\mathrm{Fc}$ blocking by a specific protein block (Novocastra, Leica Biosystems) were performed.

For immunostaining, glioma sections were probed with the following primary antibodies overnight at $4^{\circ} \mathrm{C}$ : rabbit antihuman $\mathrm{C} 1 \mathrm{q}$ polyclonal antibody (1:500; Dako), rabbit antihuman C3d polyclonal antibody (1:100; Cell Marque) and rabbit anti-human C4d polyclonal antibody (1:100; Cell Marque). Antibody-Antigen recognition was detected using Novolink Polymer Detection Systems (Novocastra Leica Biosystems, Newcastle) and employing the high sensitivity AEC (3-Amino9-Ethylcarbazole) as chromogen. Slides were counterstained with Harris Haematoxylin (Novocastra, Ltd) and images were collected using a Leica DFC320 digital camera (Leica Microsystems, Wetzlar, Germany).

For double immunostaining experiments, tissue sections were incubated overnight at $4^{\circ} \mathrm{C}$ with the following primary antibodies: rabbit anti-human $\mathrm{C} 1 \mathrm{q}$ polyclonal antibody (1:500, Dako), mouse anti-human CD68 (1:50, Clone KP1, Dako) and anti-human CD163 monoclonal antibodies (1:100, Clone 10D6, Leica Biosystems). The following secondary antibodies were used: goat anti-rabbit conjugated to Alexa Fluor 488, and goat anti-mouse conjugated to Alexa Fluor 568 (Life Technologies). Nuclei were counter-stained with DAPI $\left(4^{\prime}, 6-\right.$ diamidin-2-fenilindolo). All the sections were analyzed under Zeiss Axio Scope A1 optical microscope (Zeiss, Germany) and microphotographs were collected using an Axiocam 503 Color digital camera with the ZEN2 imaging software (Zeiss Germany).

\section{RESULTS}

\section{Bioinformatics Analysis Reveals Higher mRNA Levels of C1q $A, B$, and $C$ Chains in Gliomas}

We initially compared the mRNA levels of the three chains of human $C 1 q(A, B$, and $C)$ in normal brain and gliomas using the Oncomine platform. From the analysis performed on several datasets such as Sun's, French's, TCGA's, Rickman's, Bredel 2's, and Liang's, a significantly higher mRNA expression levels for $C 1 q A, C 1 q B$, and $C 1 q C$ chains were detected in gliomas (different histotypes and grades) as compared to normal brain tissue (Figure 1, $P<0.05$; Table 2).

We then took advantage of the UALCAN tool to carry out bioinformatics analysis on $C 1 q A, C 1 q B$, and $C 1 q C$ mRNA expression levels according to TCGA database. UALCAN tool considers LLGs, grade-II and -III, and high-grade gliomas (HGGs) only grade-IV (or GBM) while the World Health Organization (WHO) considers as LLGs, grade-I and -II, and HGGs as grade-III and -IV. Based on this analysis, as shown in Figure 2, a positive correlation between the mRNA expression of the three chains and the unfavorable prognosis only in LGGs (grade-II and -III) was evident, where the survival probability is indeed reduced $(P<0.05)$. By contrast, no correlation was observed between $C 1 q A, C 1 q B$, and $C 1 q C$ mRNA expression and the survival probability in GBMs (grade-IV) (Figure 2).

To further validate these results, we used the CGGA tool to inquire an independent glioma database. Based on this analysis, a positive correlation was found between the mRNA expression of the three $\mathrm{Clq}$ chains and the unfavorable prognosis in 


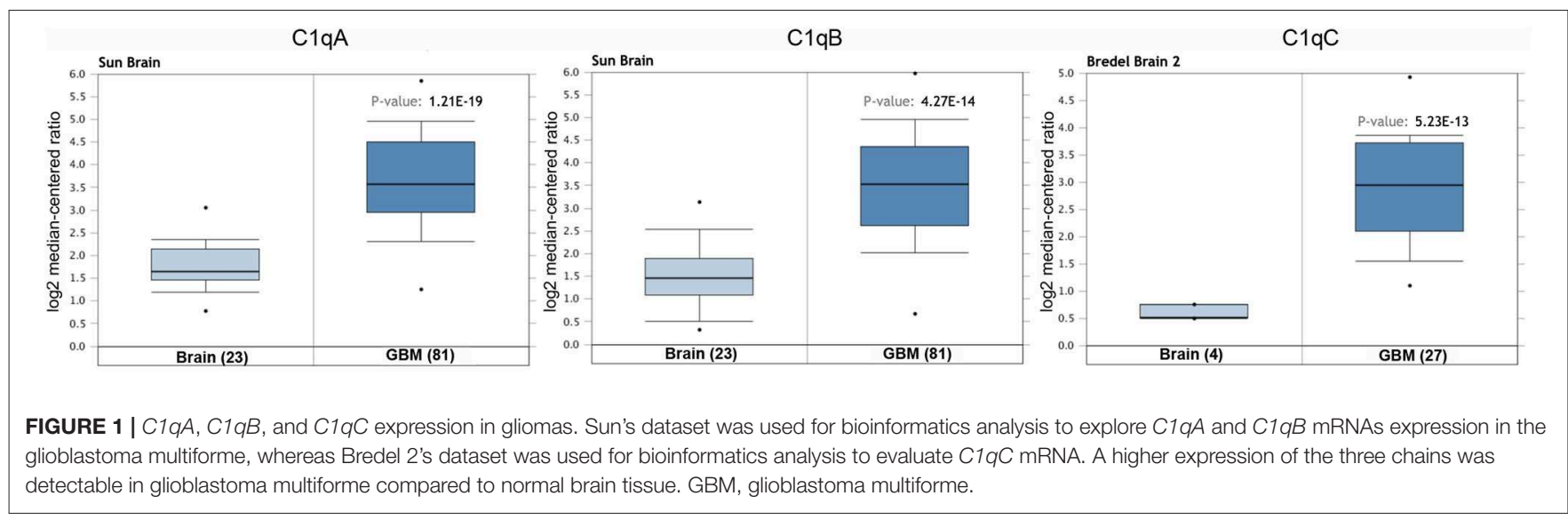

TABLE $2 \mid C 1 q A, C 1 q B$, and $C 1 q C$ expression in gliomas in the datasets used in the current study with Oncomine.

\begin{tabular}{|c|c|c|c|}
\hline Datasets & $\begin{array}{l}\text { C1q } \\
\text { chains }\end{array}$ & Brain vs. tumor & $P$-value \\
\hline \multirow[t]{2}{*}{ Sun } & C1qA & $\begin{array}{l}\text { Brain (23) vs. Anaplastic astrocytoma (grade-III) (19) } \\
\text { Brain (23) vs. Glioblastoma multiforme (grade-IV) } \\
\text { (81) } \\
\text { Brain (23) vs. Anaplastic astrocytoma (grade-IIII) (19) }\end{array}$ & $\begin{array}{c}3.59 \mathrm{E}-6 \\
1.21 \mathrm{E}-19 \\
5.22 \mathrm{E}-6\end{array}$ \\
\hline & $C 1 q B$ & $\begin{array}{l}\text { Brain (23) vs. Glioblastoma multiforme } \\
\text { (grade-IV) (81) }\end{array}$ & 4.27E-14 \\
\hline \multirow[t]{2}{*}{ French } & C1qA & $\begin{array}{l}\text { Brain (6) vs. Anaplastic oligoastrocytoma (grade-III) } \\
\text { (4) } \\
\text { Brain (6) vs. Anaplastic oligodendroglioma (grade-III) } \\
(23)\end{array}$ & $\begin{array}{l}8.89 \mathrm{E}-4 \\
3.59 \mathrm{E}-6\end{array}$ \\
\hline & $C 1 q B$ & $\begin{array}{l}\text { Brain (6) vs. Anaplastic oligoastrocytoma } \\
\text { (grade-III) (4) }\end{array}$ & 5.89E-4 \\
\hline \multirow[t]{2}{*}{ TCGA } & C1qA & $\begin{array}{l}\text { Brain (10) vs. Glioblastoma multiforme (grade-IV) } \\
\text { (542) }\end{array}$ & 1.63E-8 \\
\hline & C1qB & $\begin{array}{l}\text { Brain (10) vs. Glioblastoma multiforme (grade-IV) } \\
(542)\end{array}$ & $3.45 \mathrm{E}-4$ \\
\hline Richman & $C 1 q B$ & $\begin{array}{l}\text { Temporal lobe (10) vs. Astrocytoma (different grade) } \\
\text { (45) }\end{array}$ & 7.87E-4 \\
\hline Bredel 2 & $C 1 q C$ & Brain (4) vs. Glioblastoma multiforme (grade-IV) (28) & $5.23 \mathrm{E}-13$ \\
\hline Liang & C1qC & Brain (2) vs. Glioblastoma multiforme (grade-IV) (30) & $2.12 \mathrm{E}-4$ \\
\hline
\end{tabular}

n, samples number.

all WHO grade of gliomas, where the survival probability is indeed reduced $(P<0.05)$ (Figure 3, lowest panels). A similar unfavorable prognostic effect was detected in grade-III gliomas $(P<0.05)$ while no correlation was observed between $C 1 q A$, $C 1 q B$, and $C 1 q C$ mRNA expression and the survival probability in gliomas grade-II (Figure 3). In contrast to UALCAN analysis, a negative prognostic effect was underscored in GBMs $(P<0.05)$ (Figure 3).

\section{Low- and High-Grade Gliomas Abundantly Express C1q Protein}

We investigated the presence and the distribution of $\mathrm{Clq}$ in several glioma samples of different grades. As shown in Figure 4, a high expression level of C1q was observed both in LGGs as well as GBM.

Within the tumor-associated microenvironment, Clq was mainly expressed by monocytoid cells, suggestive of tumor associated macrophages (arrow heads) scattered among the neoplastic cells, which show an increased density around the intra-tumoral necrotic foci (Figure 4C). Moreover, the presence of $\mathrm{Clq}$ was detected in association with the vascular stroma; in GBM, it was also expressed in the vascular endothelial cells (Figures 4E,F). C1q deposition was not associated with complement activation, occurring either via the classical or the other pathways, since we failed to observe any C3d and C4d immunoreactivity in our glioma’s specimens (Figure 5).

\section{Infiltrating M2 Macrophages Are Likely Source of C1q in Gliomas}

To further characterize the cell type infiltrating the tumor and actively involved in C1q synthesis, immunocytochemical experiments were performed on gliomas specimens via staining for $\mathrm{C} 1 \mathrm{q}$ and $\mathrm{CD} 68$, a specific marker for monocyte/macrophage cell types. A clear co-localization of C1q/CD68 immunoreactivity was detected in both low- and high-grade gliomas (Figure 6), thus identifying the macrophages infiltrating glioma tumor as the main source of local C1q synthesis and secretion. Double labeling experiments were also performed using an anti-CD163, a specific marker for the tumor-promoting M2-polarized macrophages (Figure 7). Under these conditions, it appeared that not only the number of CD163 positive cells, but also the CD163 expression level itself, were increased in high-grade gliomas as compared to low-grade ones. Most of the C1q labeled cells colocalized with CD163, even though a small fraction of infiltrating cells were solely expressing $\mathrm{Clq}$, suggesting that they may correspond to the CD68/C1q co-expressing M1-type of macrophages.

\section{DISCUSSION}

Glial tumors, also called gliomas, are the most prevalent form of adult brain tumor, accounting for nearly $80 \%$ of all brain malignancies (22). Based on their histological features and expression of lineage markers, gliomas can be classified into astrocytomas, oligodendrogliomas, ependymomas, and choroid 


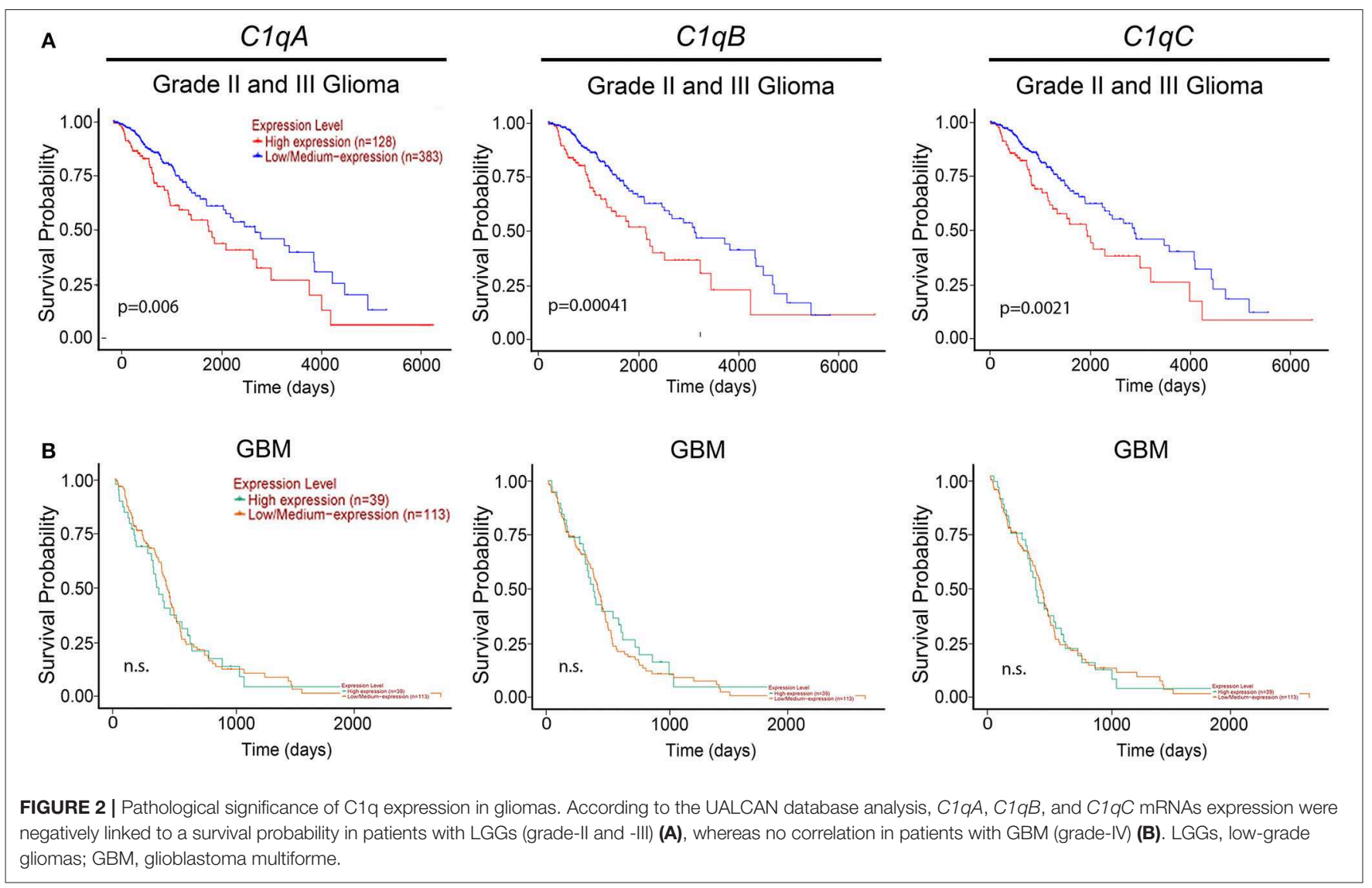

plexus tumors (29). Astrocytomas, which represent almost half of all primary brain and spinal cord tumors, may occur in the brain with a preferential localization in the cerebrum and affect mostly adults, particularly middle-aged men. In 2016, the WHO has redefined this classification scheme by introducing molecular parameters in addition to the well-established histopathological features $(20,21)$. These new guidelines have allowed classification of all pathological glial entities in four grades, according to histological parameters including nuclear atypia, mitoses, vascular proliferation, and necrosis. According to the grading system, astrocytic neoplasm can be divided into low and high-grade astrocytomas. Low-grade astrocytomas are the least malignant tumors characterized by slow growth and good prognosis being the pilocytic astrocytomas (grade-I) and diffuse astrocytomas (grade-II), the most frequent types. High-grade astrocytomas are glial tumors presenting a rapid growth with a tendency to infiltrate nearby brain tissues. They are divided into astrocytomas grade-III (anaplastic astrocytoma) and gradeIV (glioblastoma multiforme, GBM). GBM is the most aggressive and fast-growing malignancy characterized by poor clinical outcome. It can arise in the brain de novo, or it can evolve from lower-grade astrocytomas or oligodendrogliomas (29). Recent studies have begun to address the immune signature of the glioma microenvironment and its relationship with prognosis (30). The fact that C1q can be locally synthesized within the CNS and that it is involved in tumor immunology, we wanted to interrogate its relevance in the pathogenesis and prognosis of gliomas.

In the current study, we performed a bioinformatics analysis to unveil whether $\mathrm{Clq}$ could serve as a potential prognostic marker for these devastating malignancies. UALCAN queries of the TCGA and the CGGA datasets were analyzed to validate our initial result. It highlighted a significant correlation between high expression level of the three chains of the $\mathrm{C} 1 \mathrm{q}$ and poor prognosis in gliomas of diverse grade of malignancy (Figures 2, 3). In particular, while interrogating the CGGA dataset, which allowed distinction between grade-II and gradeIII gliomas, a significant correlation was established only for grade-III gliomas (Figure 3). In the TCGA dataset, significance was achieved for the so-called LGGs, which combines grade-II and -III cases (Figure 2). The main contradiction was noticed in GBMs (grade-IV gliomas), where opposite prognostic effects were underscored in relation to the dataset used (Figures 2, 3). One possible explanation for these contradictory observations may originate from the algorithm utilized by the bioinformatics web resources to define high and low expression profile of the genes. Another aspect to take into account is potential differences in the genetic and epigenetic signatures characterizing the two datasets, the TGCA mostly relying on Caucasian patients while CGGA relying on Chinese cohorts. Indeed, it has been proven that substantial variation in glioma incidence and survival are connected, to some extent, to race and 


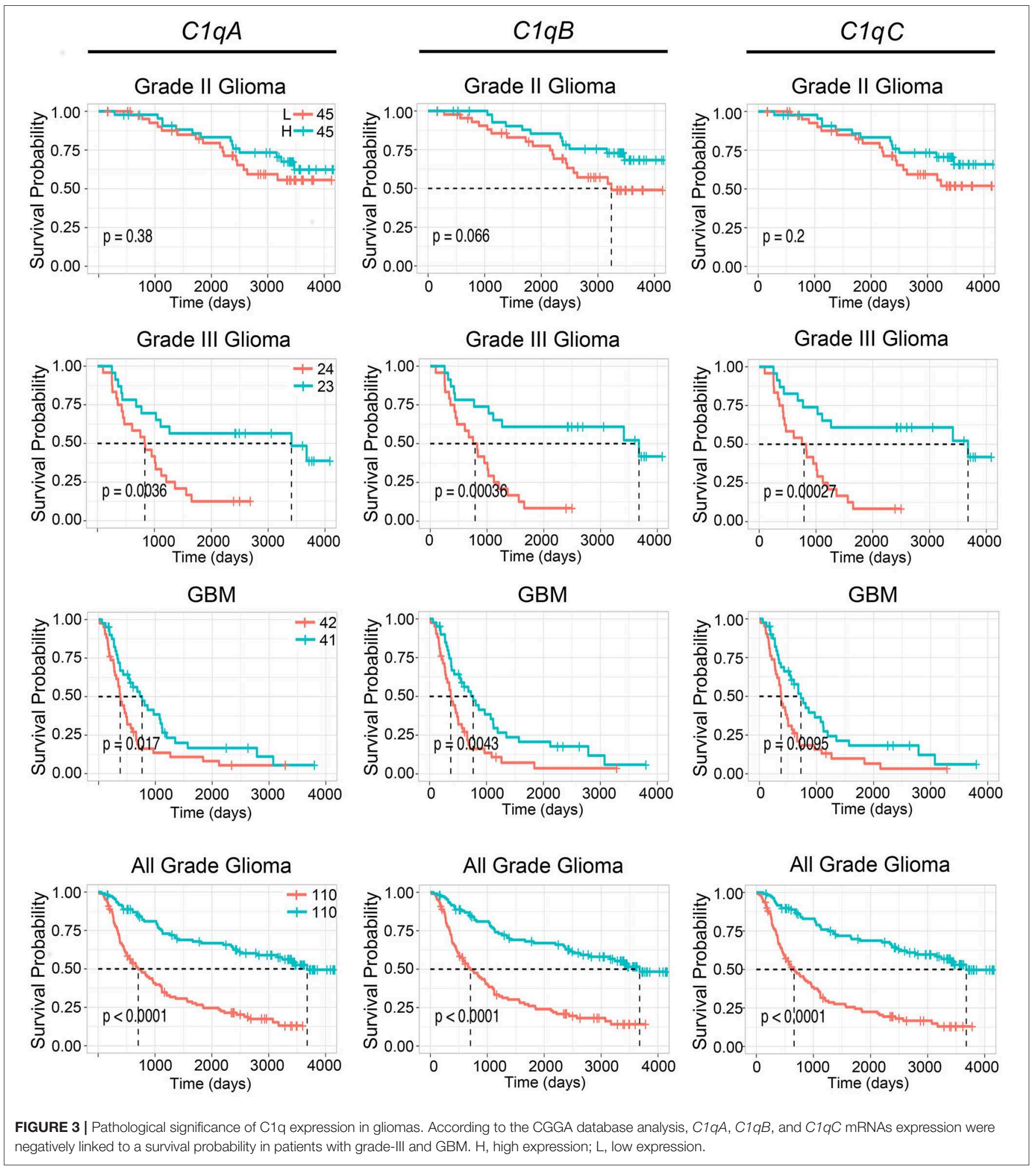

ethnicity (31). Finally, it is worth mentioning that GBM exists in two forms, primary and secondary, indistinguishable histologically, but clearly discernible clinically and in terms of molecular signatures $(29,32)$. Primary GBM is the most common form that occurs mainly in adults over 50 years of age. The genetic profile is characterized by epidermal growth factor receptor (EGFR) overexpression, phosphatase and tensin homolog (PTEN) mutation, p16 deletion, and chromosome 


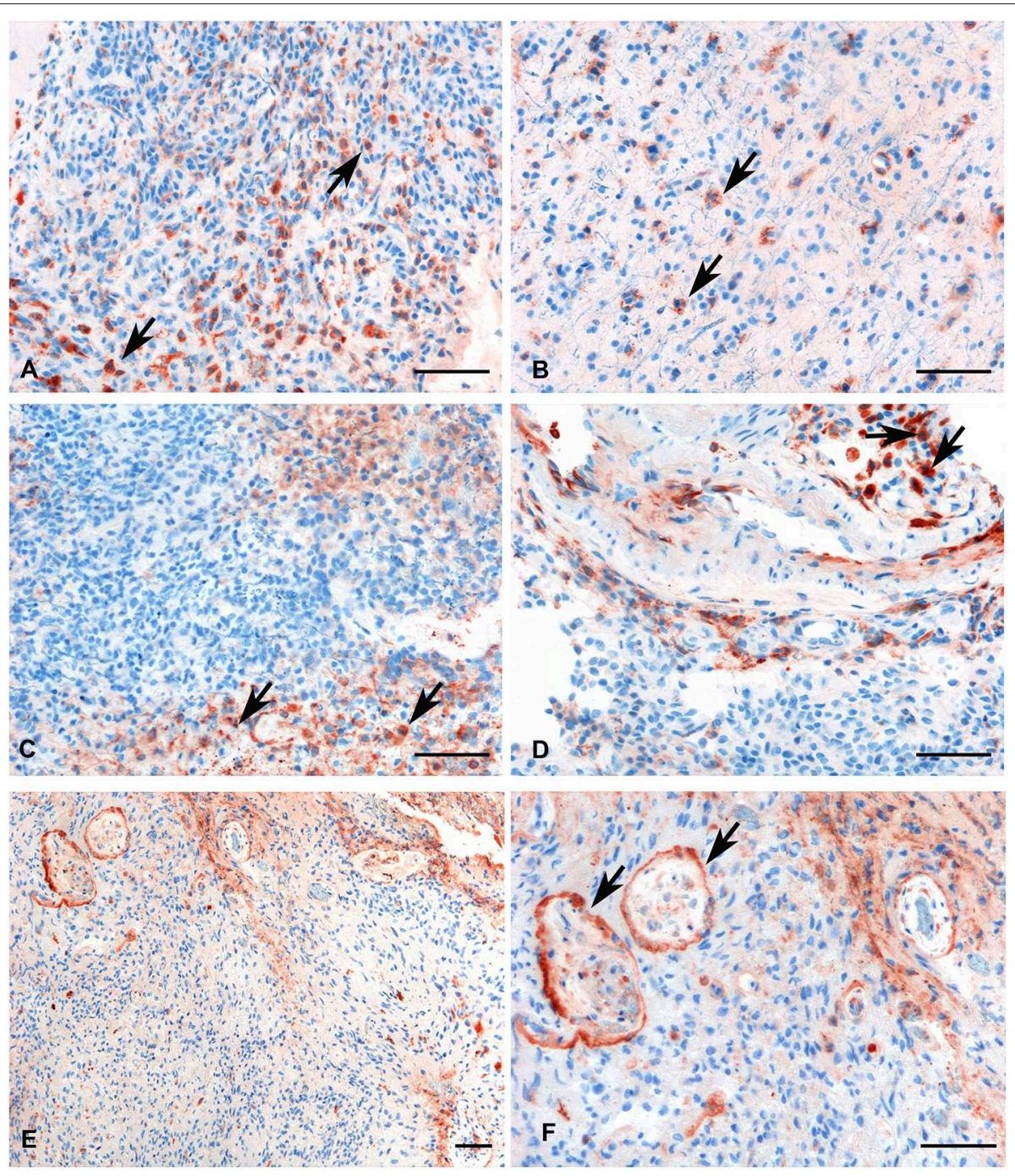

FIGURE 4 | Representative immunohistochemical analysis of C1q in low (A,B) and high grade (C,D) gliomas showing a high expression in both histotypes regardless of grade. C1q results mainly expressed by macrophages and the vascular stroma (see arrows). C1q expression in the endothelial cells is shown in panels (E,F) (see arrows, low, and high magnification). Polymer detection system with AEC (red) chromogen; scale bars, $50 \mu \mathrm{m}$.

10 loss (32). Secondary GBM derives from a malignant progression of diffuse or anaplastic astrocytomas (grade-II and III, respectively), and occurs in younger patients, characterized by p53 mutation and a reduced state of heterozygosity in tumor cells (loss of heterozygosity) on chromosome 10q (32). Both datasets used in our bioinformatics analysis did not discriminate between de novo and secondary GBM, possibly masking a significant correlation, indeed expected, in those tumors progressing from grade-III gliomas.

There is emerging evidence to confirm that $\mathrm{Clq}$ is involved in cancer pathophysiology, being an important modulator of inflammation and cytokine/chemokine/growth factor secretion. What is still quite puzzling is whether and when $\mathrm{Clq}$ is protective against or supportive of cancer progression. On one hand, $\mathrm{Clq}$ can be detrimental to cancer cell viability via its cell lytic, anaphylatoxin, and opsonin effector mechanisms
(33). Alternatively, Clq can exert tumor-promoting functions which are independent of the classical pathway activation. As observed for other types of cancers $(10,15), \mathrm{Clq}$ deposition in gliomas seems not to be correlated with complement activation as we were unable to detect the complement split products C3d and C4d in the cohort of tumor specimens tested $(n=$ 5). It is worth noting that our conclusions are based on the use of only five patients' tissue samples that were subjected to immunohistochemistry. This is in contrast to other cancers such as renal and lung cancers, where C4d deposition has been reported $(34,35)$. The mechanisms underlying such binary features of $\mathrm{Clq}$ are likely to be shaped by the type of cancer cells, nature and extent of infiltrating immune cells and their ability to synthesize locally C1q (and/or other complement components), and most crucially, the biochemical nature of the tumor microenvironment. 


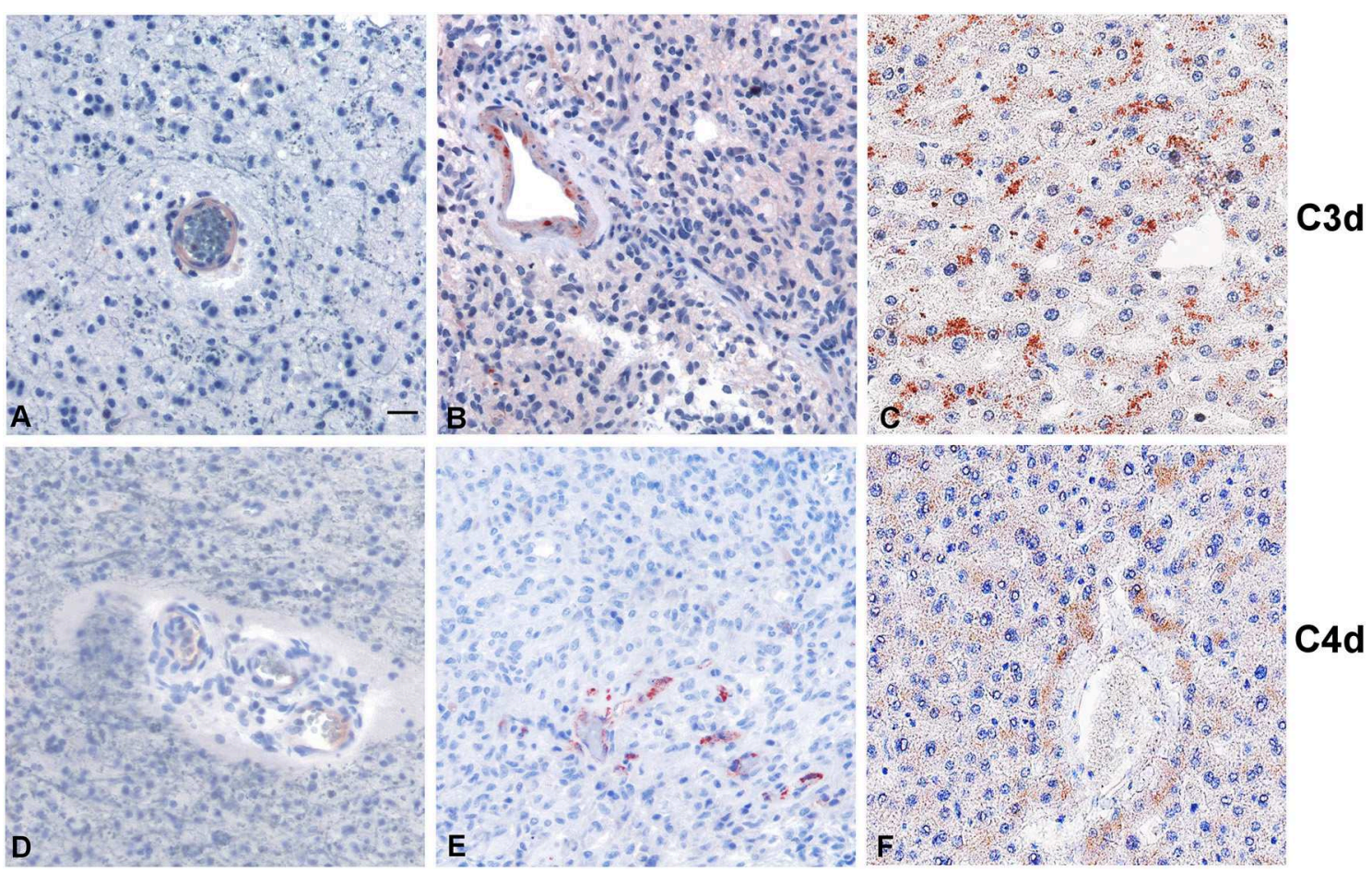

FIGURE 5 | Representative immunohistochemical analysis of C3d and C4d complement activation products in low grade (A,D) and high grade (B,E) gliomas. C3d and C4d specific staining is segregated in the blood vessels, while the tumor tissues are negative. Liver staining (C,F) represents the antigen (tissue) control. Polymer detection system with AEC (red) chromogen; scale bars, $50 \mu \mathrm{m}$.
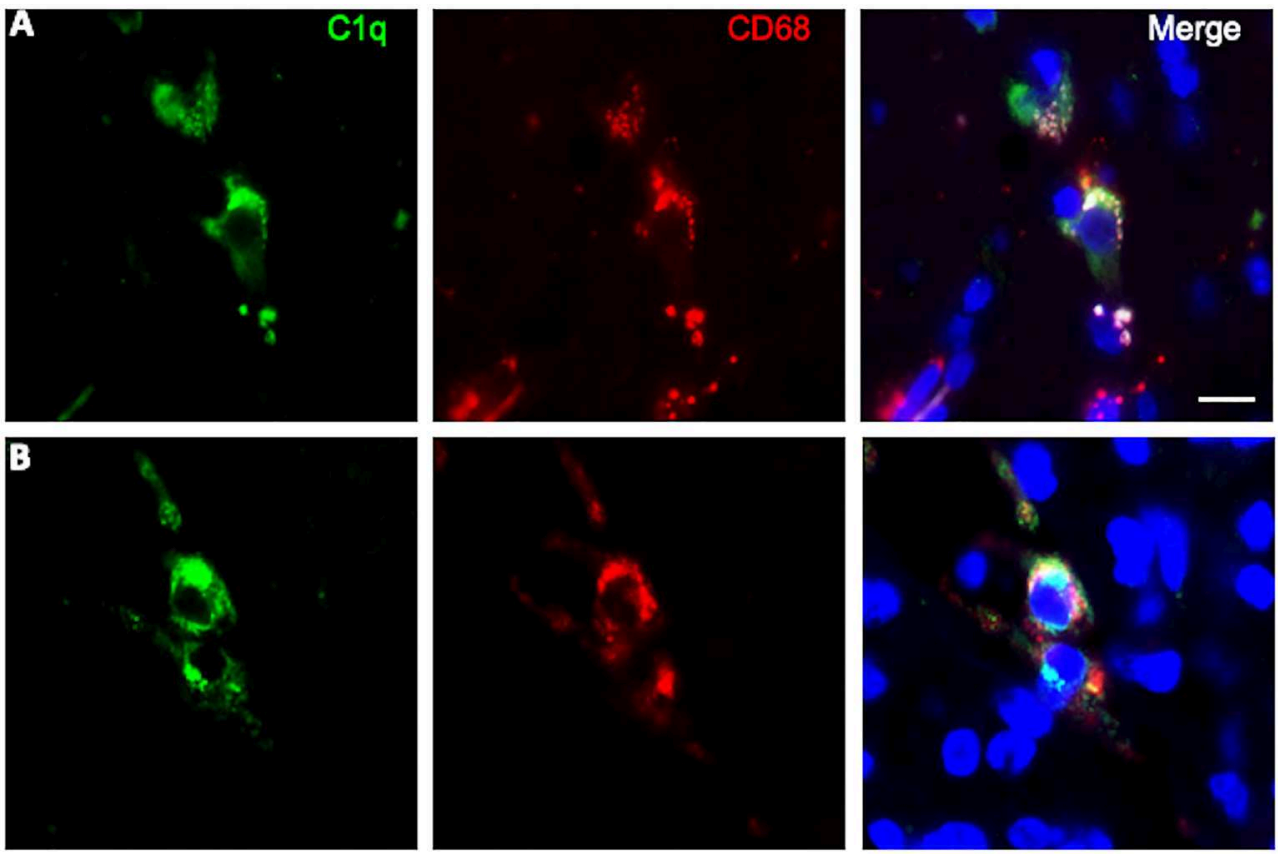

FIGURE 6 | Representative microphotographs of double immunofluorescence for C1q (green signal) and CD68 (red signal) in FFPE sections of low (A) and high (B) grade gliomas confirming the macrophage nature of $\mathrm{C} 1 \mathrm{q}$ expressing monocytoid elements. The cell nuclei were stained with DAPI; scale bars, $10 \mu \mathrm{m}$. 

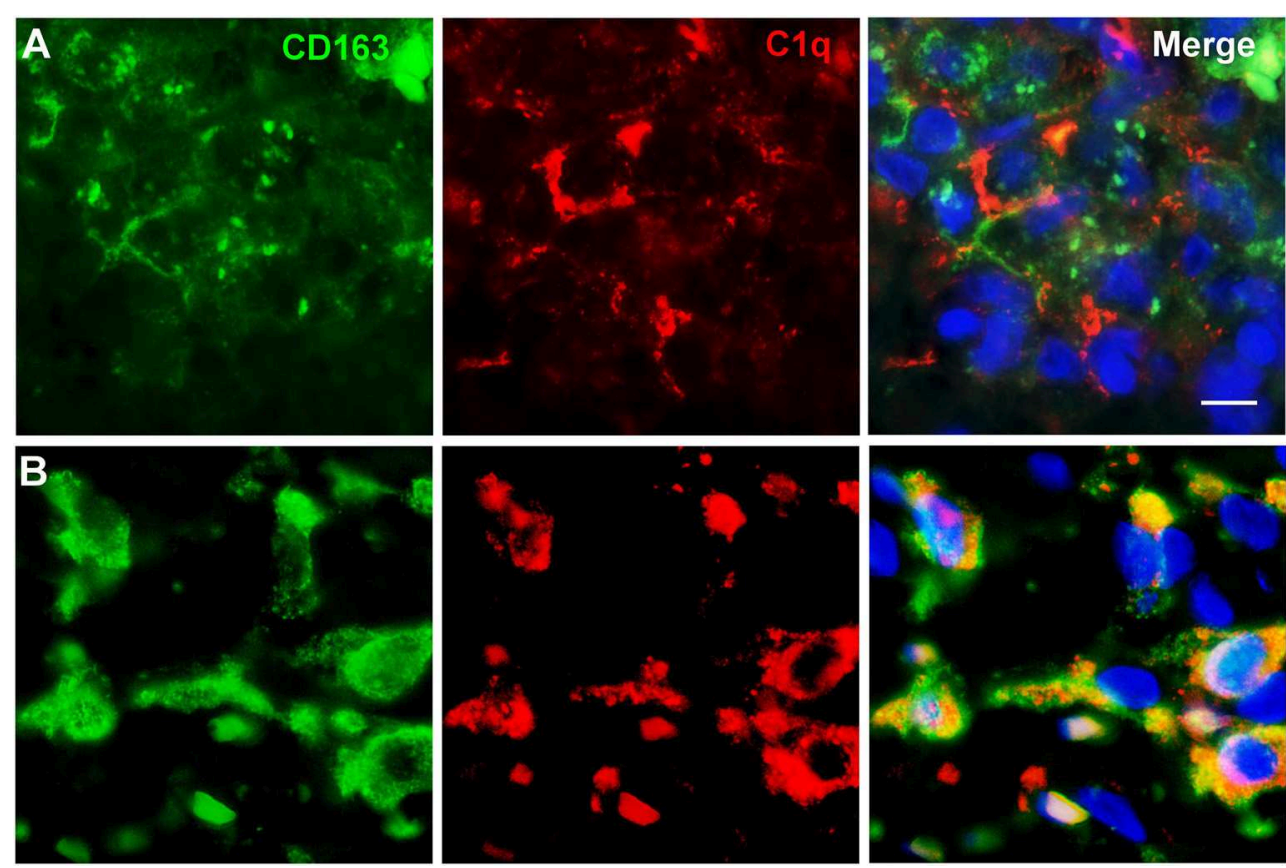

FIGURE 7 | Representative microphotographs of double immunofluorescence for C1q (red signal) and CD163 (green signal) in FFPE sections of low (A) and high (B) grade gliomas confirming the M2-polarization of macrophages expressing C1q. The cell nuclei were stained with DAPI; scale bars, $10 \mu \mathrm{m}$.

In the brain, complement components, including $\mathrm{Clq}$, can be locally produced by resident neurons and glial cells, microglia and astrocytes being the major producers $(19,36)$. Our immunocytochemical data demonstrated that CD68 and CD163 positive infiltrating cells represent the cell types actively synthesizing $\mathrm{Clq}$ in the tumor microenvironment (Figures 6, 7). CD68 expression is characteristic of tumor-associated macrophages, whose enrichment in glioma has been associated with poor prognosis (37). CD163 identify the M2-polarized macrophages, which are highly versatile cells known to influence multiple steps in tumor development and invasiveness along with angiogenesis and immunosuppression (38).

These cells in the brain are derived from two different sources: resident microglia and monocytes/macrophages that enter the brain from bone marrow. Even though it is quite difficult to distinguish between these two different cell types due to the lack of available definitive markers, they are recruited by the tumor microenvironment via several gliomas derived chemokines that also contribute to their polarization from a tumor-suppressive to a tumorpromoting phenotype (39). It is interesting to note that $\mathrm{C} 1 \mathrm{q}$ has been shown to enhance the secretion and action of these chemokines $(40,41)$. Therefore, C1q produced and released by microglia/macrophage cells, is expected to promote immunosuppression, thus favoring glioma cell proliferation. This is consistent with our observation linking the expression of the three chains of human $\mathrm{Clq}$ with an unfavorable prognosis in grade-II and -III gliomas (LGGs) in TCGA dataset and in grade-III gliomas in CGGA dataset, respectively (Figures 2, 3).
We also observed a high degree of deposition of $\mathrm{C} 1 \mathrm{q}$ in the perivascular stroma as well as on endothelial cells belonging to the tumor vasculature. Developing gliomas require an increased nutrient supply and hence trigger neovascularization via the release of angiogenetic factors by cancer cells. C1q itself promotes angiogenesis through its globular heads (7). Massive angiogenesis is induced by regions of hypoxia within the tumor that are not only loci generating damage-associated molecular patterns (DAMPs), recognized by $\mathrm{Clq}$ but also privileged sites for glioma stem-like cell (GSC) settlement (42). These cells are quite dangerous since they maintain tumor growth through self-renewal amidst a supportive microenvironment. C1q, in this context, has been shown to participate in GSC maintenance and expansion via the activation of the canonical Wnt signaling cascade through its binding to Frizzled-receptor (43).

Our bioinformatics study, based solely on mRNA expression dataset and therefore further requiring a validation at the protein level, underlines how complex, multifaceted and yet feebly understood is the differential role of $\mathrm{Clq}$ in tumor progression or suppression. In conclusion, $\mathrm{Clq}$ plays a fundamental role in the pathogenesis of gliomas, but further investigation is required for its use in the clinic as a prognostic marker.

\section{DATA AVAILABILITY STATEMENT}

Publicly available datasets were analyzed in this study. This data can be found here: TCGA-GBM, TCGA-LGG (https://tcga-data. nci.nih.gov/tcga/), and CGGA (http://www.cgga.org.cn). 


\section{ETHICS STATEMENT}

This study was carried out in accordance with the recommendations of government guidelines, and approved by the CEUR (Comitato Etico Unico Regionale, FVG, Italy; number 34/2016). All subjects gave written informed consent in accordance with the Declaration of Helsinki.

\section{AUTHOR CONTRIBUTIONS}

AMan, PZ, and RB: conceptualization and writing-original draft preparation. AMar and BB: methodology. AMan: software and visualization. DB: validation. FZ: formal analysis. $\mathrm{BB}$ and $\mathrm{DB}$ :

\section{REFERENCES}

1. Lu J, Kishore U. C1 complex: an adaptable proteolytic module for complement and non-complement functions. Front Immunol. (2017) 8:592. doi: 10.3389/fimmu.2017.00592

2. Kishore U, Reid KB. C1q: structure, function, and receptors. Immunopharmacology. (2000) 49:159-70. doi: 10.1016/S0162-3109(00)80301-X

3. Sellar GC, Cockburn D, Reid KB. Localization of the gene cluster encoding the $\mathrm{A}, \mathrm{B}$, and C chains of human C1q to 1p34.1-1p36.3. Immunogenetics. (1992) 35:214-6. doi: 10.1007/BF00185116

4. Reid KB, Porter RR. Subunit composition and structure of subcomponent C1q of the first component of human complement. Biochem J. (1976) 155:19-23. doi: 10.1042/bj1550019

5. Kishore U, Gaboriaud C, Waters P, Shrive AK, Greenhough TJ, Reid KB, et al. $\mathrm{C} 1 \mathrm{q}$ and tumor necrosis factor superfamily: modularity and versatility. Trends Immunol. (2004) 25:551-61. doi: 10.1016/j.it.2004.08.006

6. Agostinis C, Bulla R, Tripodo C, Gismondi A, Stabile H, Bossi F, et al. An alternative role of $\mathrm{Cl} q$ in cell migration and tissue remodeling: contribution to trophoblast invasion and placental development. J Immunol. (2010) 185:44209. doi: 10.4049/jimmunol.0903215

7. Bossi F, Tripodo C, Rizzi L, Bulla R, Agostinis C, Guarnotta C, et al. C1q as a unique player in angiogenesis with therapeutic implication in wound healing. Proc Natl Acad Sci USA. (2014) 111:4209-14. doi: 10.1073/pnas.1311968111

8. Son M, Diamond B, Santiago-Schwarz F. Fundamental role of $\mathrm{C} 1 \mathrm{q}$ in autoimmunity and inflammation. Immunol Res. (2015) 63:101-6. doi: 10.1007/s12026-015-8705-6

9. Ghebrehiwet B, Hosszu KK, Valentino A, Peerschke EI. The C1q family of proteins: insights into the emerging non-traditional functions. Front Immunol. (2012) 3:52. doi: 10.3389/fimmu.2012.00052

10. Bulla R, Tripodo C, Rami D, Ling GS, Agostinis C, Guarnotta C, et al. $\mathrm{C} 1 \mathrm{q}$ acts in the tumour microenvironment as a cancer-promoting factor independently of complement activation. Nat Commun. (2016) 7:10346. doi: 10.1038/ncomms10346

11. Agostinis C, Vidergar R, Belmonte B, Mangogna A, Amadio L, Geri P, et al. Complement protein $\mathrm{C} 1 \mathrm{q}$ binds to hyaluronic acid in the malignant pleural mesothelioma microenvironment and promotes tumor growth. Front Immunol. (2017) 8:1559. doi: 10.3389/fimmu.2017.01559

12. Hong Q, Sze CI, Lin SR, Lee MH, He RY, Schultz L, et al. Complement C1q activates tumor suppressor WWOX to induce apoptosis in prostate cancer cells. PLoS ONE. (2009) 4:e5755. doi: 10.1371/journal.pone.0005755

13. Kaur A, Sultan SH, Murugaiah V, Pathan AA, Alhamlan FS, Karteris $\mathrm{E}$, et al. Human $\mathrm{Clq}$ induces apoptosis in an ovarian cancer cell line via tumor necrosis factor pathway. Front Immunol. (2016) 7:599. doi: 10.3389/fimmu.2016.00599

14. Bandini S, Macagno M, Hysi A, Lanzardo S, Conti L, Bello A, et al. The noninflammatory role of $\mathrm{Clq}$ during Her2/neu-driven mammary carcinogenesis. Oncoimmunology. (2016) 5:e1253653. doi: 10.1080/2162402X.2016.1253653 investigation. AMan, VR, DI, and FZ: resources. CA: data curation. PZ, CA, and UK: writing-review and editing. RB: supervision and project administration.

\section{FUNDING}

This work was supported by grant from POR FESR FVG 20142020 TICheP to RB.

\section{ACKNOWLEDGMENTS}

The authors would like to thank Alessandro Gulino for the immunohistochemical analysis.

15. Mangogna A, Agostinis C, Bonazza D, Belmonte B, Zacchi P, Zito G, et al. Is the complement protein $\mathrm{C} 1 \mathrm{q}$ a pro- or anti-tumorigenic factor? bioinformatics analysis involving human carcinomas. Front Immunol. (2019) 10:865. doi: 10.3389/fimmu.2019.00865

16. Stevens B, Allen NJ, Vazquez LE, Howell GR, Christopherson KS, Nouri N, et al. The classical complement cascade mediates CNS synapse elimination. Cell. (2007) 131:1164-78. doi: 10.1016/j.cell.2007.10.036

17. Pisalyaput $\mathrm{K}$, Tenner AJ. Complement component $\mathrm{C} 1 \mathrm{q}$ inhibits betaamyloid- and serum amyloid P-induced neurotoxicity via caspase- and calpain-independent mechanisms. J Neurochem. (2008) 104:696-707. doi: 10.1111/j.1471-4159.2007.05012.x

18. Cho K. Emerging roles of complement protein $\mathrm{C} 1 \mathrm{q}$ in neurodegeneration. Aging Dis. (2019) 10:652-63. doi: 10.14336/AD.2019.0118

19. Bouwens van der Vlis TAM, Kros JM, Mustafa DAM, van Wijck RTA, Ackermans L, van Hagen PM, et al. The complement system in glioblastoma multiforme. Acta Neuropathol Commun. (2018) 6:91. doi: 10.1186/s40478-018-0591-4

20. Louis DN, Perry A, Reifenberger G, von Deimling A, Figarella-Branger D, Cavenee WK, et al. The 2016 World Health Organization classification of tumors of the central nervous system: a summary. Acta Neuropathol. (2016) 131:803-20. doi: 10.1007/s00401-016-1545-1

21. Louis DN, Ohgaki H, Wiestler OD, Cavenee WK, World Health Organization, International Agency for Research on Cancer. WHO Classification of Tumours of the Central Nervous System, Revised. 4th ed. Lyon: International Agency For Research on Cancer (2016). p. 408.

22. Ostrom QT, Gittleman H, Liao P, Vecchione-Koval T, Wolinsky Y, Kruchko C, et al. CBTRUS Statistical Report: primary brain and other central nervous system tumors diagnosed in the United States in 2010-2014. Neuro Oncol. (2017) 19:v1-88. doi: 10.1093/neuonc/nox158

23. Bouwens TA, Trouw LA, Veerhuis R, Dirven CM, Lamfers ML, Al-Khawaja H. Complement activation in Glioblastoma multiforme pathophysiology: evidence from serum levels and presence of complement activation products in tumor tissue. J Neuroimmunol. (2015) 278:271-6. doi: 10.1016/j.jneuroim.2014.11.016

24. Rhodes DR, Kalyana-Sundaram S, Mahavisno V, Varambally R, Yu J, Briggs $\mathrm{BB}$, et al. Oncomine 3.0: genes, pathways, and networks in a collection of 18,000 cancer gene expression profiles. Neoplasia. (2007) 9:166-80. doi: $10.1593 /$ neo.07112

25. Rhodes DR, Yu J, Shanker K, Deshpande N, Varambally R, Ghosh D, et al. ONCOMINE: a cancer microarray database and integrated datamining platform. Neoplasia. (2004) 6:1-6. doi: 10.1016/S1476-5586(04)8 0047-2

26. Chandrashekar DS, Bashel B, Balasubramanya SAH, Creighton CJ, PonceRodriguez I, Chakravarthi B, et al. UALCAN: a portal for facilitating tumor subgroup gene expression and survival analyses. Neoplasia. (2017) 19:649-58. doi: 10.1016/j.neo.2017.05.002

27. Yan W, Zhang W, You G, Zhang J, Han L, Bao Z, et al. Molecular classification of gliomas based on whole genome gene expression: a systematic report of 225 
samples from the Chinese Glioma Cooperative Group. Neuro Oncol. (2012) 14:1432-40. doi: 10.1093/neuonc/nos263

28. Mangogna A, Belmonte B, Agostinis C, Ricci G, Gulino A, Ferrara I, et al. Pathological significance and prognostic value of surfactant protein $\mathrm{D}$ in cancer. Front Immunol. (2018) 9:1748. doi: 10.3389/fimmu.2018.01748

29. Louis DN, International Agency for Research on Cancer, World Health Organization. WHO Classification of Tumours of the Central Nervous System. Lyon: International Agency for Research on Cancer (2007). p. 309.

30. Zhang B, Shen R, Cheng S, Feng L. Immune microenvironments differ in immune characteristics and outcome of glioblastoma multiforme. Cancer Med. (2019) 8:2897-907. doi: 10.1002/cam4.2192

31. Ostrom QT, Cote DJ, Ascha M, Kruchko C, Barnholtz-Sloan JS. Adult glioma incidence and survival by race or ethnicity in the United States from 2000 to 2014. JAMA Oncol. (2018) 4:1254-62. doi: 10.1001/jamaoncol.2018.1789

32. Ohgaki H, Kleihues P. Genetic pathways to primary and secondary glioblastoma. Am J Pathol. (2007) 170:1445-53. doi: 10.2353/ajpath.2007.070011

33. Reis ES, Mastellos DC, Ricklin D, Mantovani A, Lambris JD. Complement in cancer: untangling an intricate relationship. Nat Rev Immunol. (2018) 18:5-18. doi: 10.1038/nri.2017.97

34. Roumenina LT, Daugan MV, Noe R, Petitprez F, Vano YA, SanchezSalas R, et al. Tumor cells hijack macrophage-produced complement Clq to promote tumor growth. Cancer Immunol Res. (2019) 7:1091-105. doi: 10.1158/2326-6066.CIR-18-0891

35. Ajona D, Pajares MJ, Corrales L, Perez-Gracia JL, Agorreta J, Lozano MD, et al. Investigation of complement activation product c4d as a diagnostic and prognostic biomarker for lung cancer. J Natl Cancer Inst. (2013) 105:1385-93. doi: $10.1093 /$ jnci/djt205

36. Veerhuis R, Nielsen HM, Tenner AJ. Complement in the brain. Mol Immunol. (2011) 48:1592-603. doi: 10.1016/j.molimm.2011.04.003

37. Wang L, Zhang C, Zhang Z, Han B, Shen Z, Li L, et al. Specific clinical and immune features of CD68 in glioma via 1,024 samples. Cancer Manag Res. (2018) 10:6409-19. doi: 10.2147/CMAR.S183293
38. Galdiero MR, Garlanda C, Jaillon S, Marone G, Mantovani A. Tumor associated macrophages and neutrophils in tumor progression. J Cell Physiol. (2013) 228:1404-12. doi: 10.1002/jcp.24260

39. Hambardzumyan D, Gutmann DH, Kettenmann H. The role of microglia and macrophages in glioma maintenance and progression. Nat Neurosci. (2016) 19:20-7. doi: 10.1038/nn.4185

40. Peerschke EI, Ghebrehiwet B. $\mathrm{cC} 1 \mathrm{qR} / \mathrm{CR}$ and $\mathrm{gClqR} / \mathrm{p} 33$ : observations in cancer. Mol Immunol. (2014) 61:100-9. doi: 10.1016/j.molimm.2014. 06.011

41. Qiu Y, Marquez-Curtis LA, Janowska-Wieczorek A. Mesenchymal stromal cells derived from umbilical cord blood migrate in response to complement C1q. Cytotherapy. (2012) 14:285-95. doi: 10.3109/14653249.2011. 651532

42. Lathia JD, Heddleston JM, Venere M, Rich JN. Deadly teamwork: neural cancer stem cells and the tumor microenvironment. Cell Stem Cell. (2011) 8:482-5. doi: 10.1016/j.stem.2011.04.013

43. Kalani MY, Cheshier SH, Cord BJ, Bababeygy SR, Vogel H, Weissman IL, et al. Wnt-mediated self-renewal of neural stem/progenitor cells. Proc Natl Acad Sci USA. (2008) 105:16970-5. doi: 10.1073/pnas.08086 16105

Conflict of Interest: The authors declare that the research was conducted in the absence of any commercial or financial relationships that could be construed as a potential conflict of interest.

Copyright () 2019 Mangogna, Belmonte, Agostinis, Zacchi, Iacopino, Martorana, Rodolico, Bonazza, Zanconati, Kishore and Bulla. This is an open-access article distributed under the terms of the Creative Commons Attribution License (CC BY). The use, distribution or reproduction in other forums is permitted, provided the original author(s) and the copyright owner(s) are credited and that the original publication in this journal is cited, in accordance with accepted academic practice. No use, distribution or reproduction is permitted which does not comply with these terms. 